\title{
Use of Computed Tomography Scan to Rule Out Phantom Thrombus in the Left Atrial Appendage
}

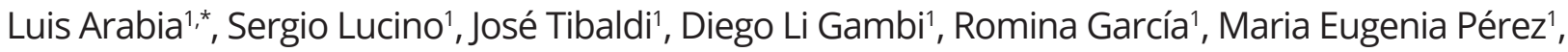
Franco Bottello ${ }^{1}$

ORCID IDs

Arabia L ${ }^{(1 D}$ https://orcid.org/0000-0003-0673-0234

Lucino S (Dttps://orcid.org/0000-0002-9041-1713

Tibaldi J ${ }^{(1)}$ https://orcid.org/0000-0003-1812-7985

García R (iD https://orcid.org/0000-0001-6447-3059

Li Gambi D (D) https://orcid.org/0000-0001-6724-9511

Pérez ME (D) https://orcid.org/0000-0002-1274-4571

Bottello F (D) https://orcid.org/0000-0001-6449-8117

\begin{abstract}
Objective: The aim of study was to evaluate the utility of CT scan with delayed acquisition protocol to exclude LAA thrombus. The occurrence of left atrial appendage (LAA) thrombus is a frequent complication of atrial fibrillation (AF) and increases the thromboembolic risk. Transesophageal echocardiography (TEE) is considered the gold standard to ensure that this chamber is thrombus-free. Multidetector computed tomography (CT) scan has some advantages, such the possibility to get 3D reconstruction and explore other structures in relationship with the LAA. However, there is a lack of specificity in case of false positive images with filling defects due to slow velocities in the LAA. Methods and Results: Thirty-four patients with suspected thrombus by a previous CT scan or transesophageal echocardiogram were included in the study. In all of patients, complete LAA filling was observed, with sensitivity, specificity and negative predictive value of 100\% to differentiate circulatory stasis from thrombus. Conclusion: Performing a CT scan with delayed acquisition protocol and in prone position are safe techniques to discard false thrombus.
\end{abstract}

KEYWORDS: Atrial fibrillation; Thrombus; Left atrial appendage; Computed tomography.

1. Instituto Oulton - Cordoba, Argentina.

*Correspondence author: luisarabi@hotmail.com

Received: 12 May 2020 | Accepted: 20 May 2020 


\section{INTRODUCTION}

The occurrence of thrombus in the left atrial appendage (LAA) is a frequent complication of atrial fibrillation (AF), increases systemic and cerebral thromboembolic risk, and is a contraindication to perform percutaneous procedures in the left atrium (LA), so it must be ruled out before performing them ${ }^{1}$.

The LAA is the source of clots in almost $90 \%$ in patients with $\mathrm{AF}^{2}$. Transesophageal echocardiography (TEE) is considered the gold standard to ensure that this chamber is thrombus-free, but it is a semiinvasive study, with different degrees of discomfort, sometimes requiring anesthetic support and, in really few cases, perforation of the esophagus have been reported ${ }^{1,3-8}$.

By the other hand, multidetector computed tomography (CT) scan has some advantages, such as the possibility to get $3 \mathrm{D}$ reconstruction and explore other structures in relationship with the LAA, but it has the risk of contrast toxicity and radiation exposure, and lack of specificity, in case of false positive images, with filling defects due to slow velocities in the LAA. Several protocols were used to increase positive and negative predictor value (PPV, NPV), such as delayed acquisitions sequences, different position of the body (prone position), and use of Hounsfield unit (HU) density ${ }^{9-23}$.

\section{PATIENTS AND METHODS}

From May 2017 to January 2020, 34 patients were enrolled., of which 26 were male, between 41-84 years old (mean age $=68$ ), referred for AF ablation and/or LAA occlusion (LAAO). All of them suspected to have a thrombus by a previous $\mathrm{CT}$ scan or transesophageal echocardiogram.

\section{Procedure}

A 64-detector-row CT scanner (Toshiba Multislice Aquilion 64; Toshiba Medical Systems, Otawara, Japan) was used from 2017 to May 2019 and, after that, an Aquilion Prime Cannon/Toshiba 80/160. Slices thickness were done every $0.5 \mathrm{~mm}$ and $0.3 \mathrm{~s}$. With Aquilion 64 system, the electrocardiogram (ECG) was simultaneously recorded for retrospective gating, and a prospective volumetric iterative reconstruction was made with the Aquilion Prime. $80-100 \mathrm{kV}, 80-150 \mathrm{ml}$ of contrast, 4,5- $5 \mathrm{mSv}$ for all the three sequences (about 1.5 each), depending of the BMI with the last MDCT scan, instead $15+/-3 \mathrm{mSv}$ with the first MDCT.

First, a conventional gated CT was done; if a filling defect in the LAA was seen, a second sequence would start three minutes later, with a second bolus of $40 \mathrm{cc}$ of contrast and delayed acquisition with six cuts every three seconds, with the ROI in ascendant aorta, and compared with LAA not only the visual impression but the HU, and a third sequence (same protocol than the first one), with the patient in prone position was done, with a low Rx exposure and contrast dose protocol.

\section{RESULTS}

Complete filling in the second and third sequence was observed in all of the patients, and AF ablation, LAA closure or both were done without thromboembolic complications neither during the procedure nor at a follow-up of 3 to 28 months. This protocol allowed us to differentiate circulatory stasis from thrombus with a sensitivity, specificity and negative predictive value of $100 \%$. 


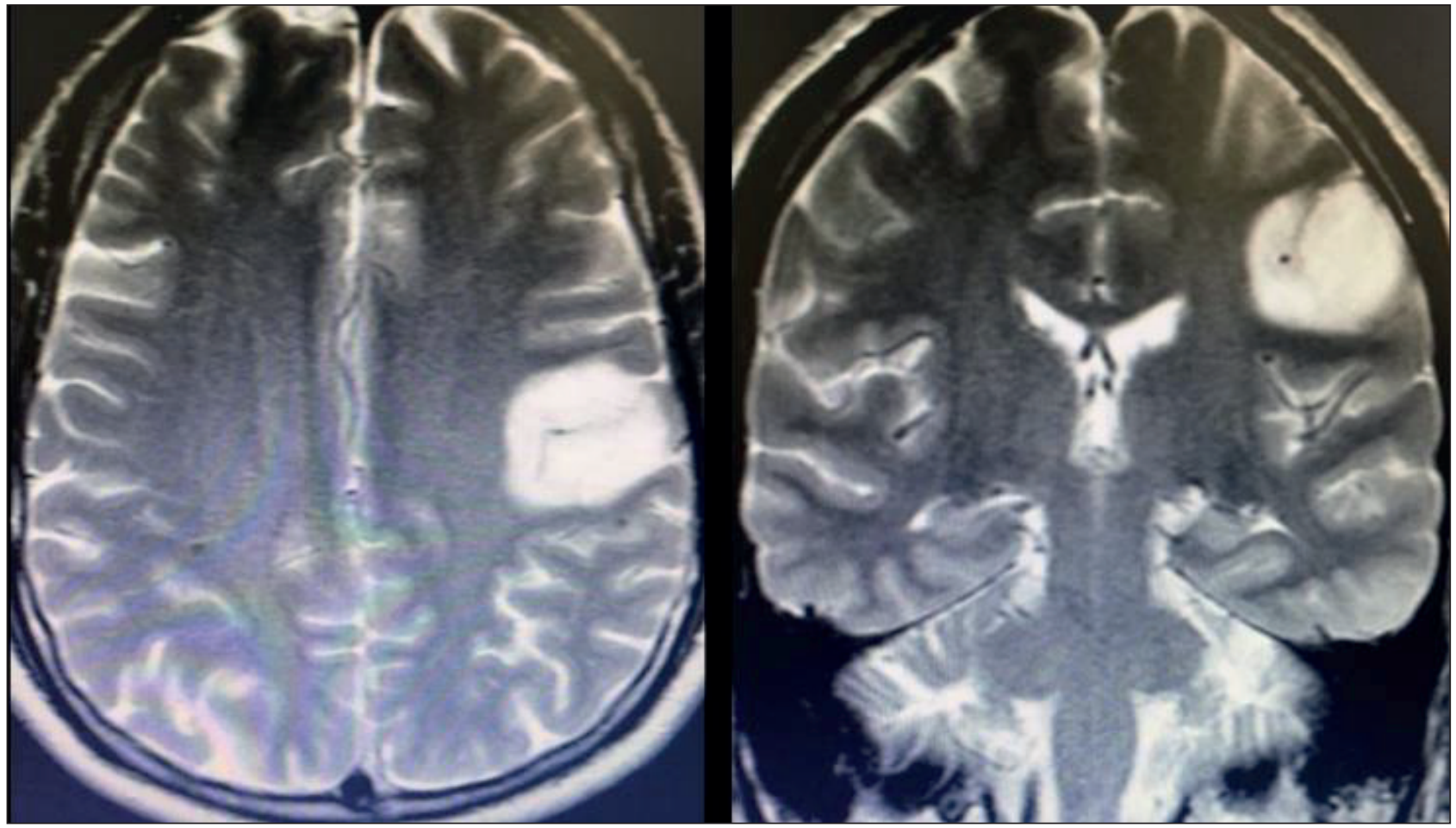

Figure 1. Magnetic resonance image from a male patient with 43 years old, permanent atrial fibrillation and restrictive cardiomyopathy. 40 minutes of symptoms onset. Next images are from the same patient performed the same day.

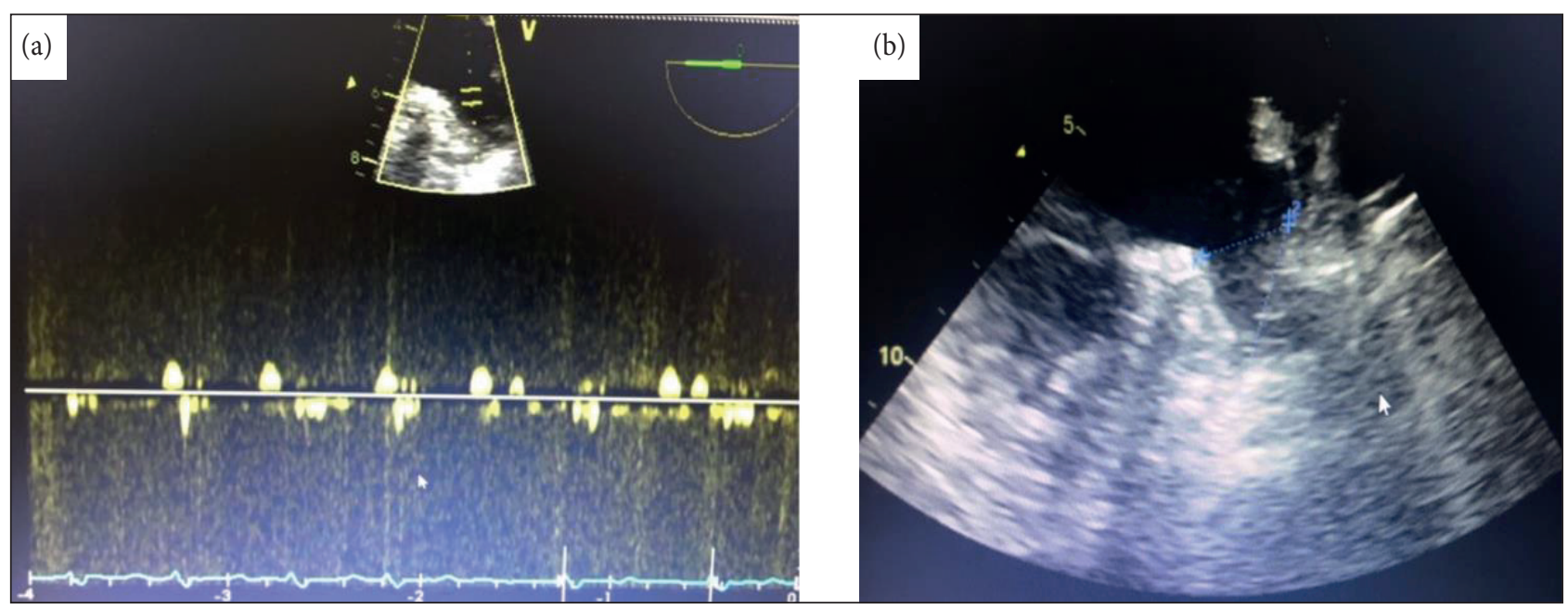

Figure 2. Transesophageal echocardiogram: (a) with Doppler velocity of left atrial appendage (LAA), (b) with "smoke", spontaneous contrast in the left atrium (LA) and LAA, and sparklier image inside LAA.
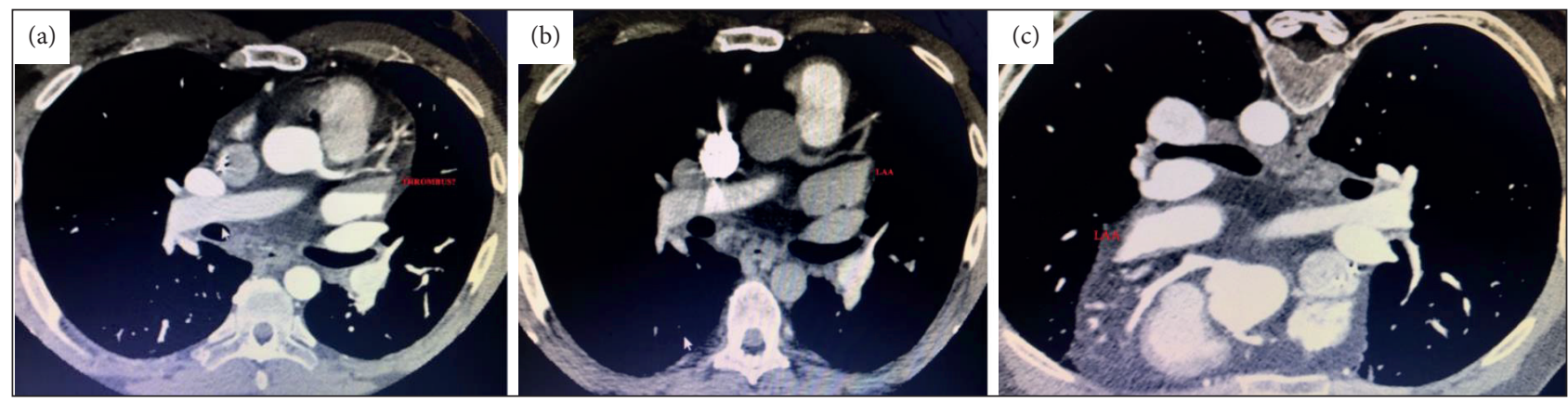

Figure 3. Computed tomography (CT): (a) first sequence with a filling defect in the LAA, (b) second delayed sequence showing complete filling of the LAA, (c) third sequence in prone position, showing complete filling of the LAA. 


\section{DISCUSSION}

Intracavitary LA/LAA filling defects can be seen due to thrombus presence or for inadequate mixing of blood and contrast, giving a false positive image of a clot. Altered flow with reduced velocities are not infrequent in patients with AF; moreover, if they have ventricular disfunction ${ }^{12,24,25}$.

A filling defect was defined as an area of low attenuation seen in the LA or LAA, different of pectinate muscles or another structure. These defects were classified in low, moderate or high risk using the homoneity of the low attenuation zone, border aspect and $\mathrm{HU}$ value, the highest risk being those homogeneous, less than $100 \mathrm{HU}$ and well-defined border; and the opposite, nonhomogeneous aspect, an indefinite border and more than $100 \mathrm{HU}$ for the low risk studies ${ }^{10,26}$.

While there are different protocols using delayed acquisitions (single vs double bolus, different times ranging from 30 to 180 seconds), when they are used, the diagnostic accuracy clearly improved, reducing the number of false positive $\mathrm{CT}^{10,13-18}$.

Studies in prone position were published to have similar PPV and NPV, such as delayed acquisition protocols ${ }^{19,21}$. This research only found one study combining both techniques ${ }^{22}$, but not in the same three steps that were used in this work if a filling defect was seen in the first scan.

The two mayor concerns about $\mathrm{CT}$ are the use of iodinate contrast agent and the amount of ionizing radiation to these patients are exposed, both can be reduced using new systems, adjusting dose and using prospective volumetric reconstruction, because the radiation source is active only in a short segment of $\mathrm{R}-\mathrm{R}$ interval, saving a big amount of the dose ${ }^{27}$.

\section{CONCLUSION}

Left atrial appendage thrombus has a severe risk of thromboembolic complications and is a contraindication to percutaneous access to the left atrium. Performing a multidetector CT scan with a delayed acquisition protocol and in prone position are safe techniques to rule out false thrombus. More studies are needed to validate these findings.

\section{ACKNOWLEDGMENTS}

Our acknowledgment to all technical workers of CT scan Department for their continuous support.

\section{REFERENCES}

1. Calkins H, Kuck KH, Cappato R, Brugada J, Camm AJ, Chen S-A, et al. 2012 HRS/EHRA/ECAS expert consensus statement on catheter and surgical ablation of atrial fibrillation: recommendations for patient selection, procedural techniques, patient management and follow-up, definitions, endpoints, and research trial design. Heart Rhythm. 2012;9(4):632-96. https://doi.org/10.1016/j. hrthm.2011.12.016

2. Allbutt C, Rolleston HD. A system of medicine. London: MacMillan; 1909.

3. Beigel R, Wunderlich NC, Ho SY, Arsanjani R, Siegel RJ. The left atrial appendage: anatomy, function, and noninvasive evaluation. JACC Cardiovasc Imaging. 2014;7(12):1251-65. https://doi.org/10.1016/j.jcmg.2014.08.009

4. Manning WJ, Weintraub RM, Waksmonski CA, Haering M, Rooney PS, Maslow AD, et al. Accuracy of transesophageal echocardiography for identifying left atrial thrombi: a prospective, intraoperative study. Ann Intern Med. 1995;123(11):817-22. https://doi. org/10.7326/0003-4819-123-11-199512010-00001 
5. Chan K-L, Cohen Gl, Sochowski RA, Baird MG. Complications of transesophageal echocardiography in ambulatory adult patients: analysis of 1500 consecutive examinations. J Am Soc Echocardiogr. 1991;4(6):577-82. https://doi.org/10.1016/S08947317(14)80216-2

6. Daniel WG, Erbel R, Kasper W, Visser CA, Engberding R, Sutherland GR, et al. Safety of transesophageal echocardiography. A multicenter survey of 10,419 examinations. Circulation. 1991;83(3):817-21 https://doi.org/10.1161/01.CIR.83.3.817

7. Kallmeyer IJ, Collard CD, Fox JA, Body SC, Shernan SK. The safety of intraoperative transesophageal echocardiography: a case series of 7200 cardiac surgical patients. Anesth Analg. 2001;92(5):1126-30. https://doi.org/10.1097/00000539-200105000-00009

8. Min JK, Spencer KT, Furlong KT, DeCara JM, Sugeng L, Ward RP, et al. Clinical features of complications from transesophageal echocardiography: a single-center case series of 10,000 consecutive examinations. J Am Soc Echocardiogr. 2005;18(9):925-9. https://doi.org/10.1016/j.echo.2005.01.034

9. Bilchick KC, Mealor A, Gonzalez J, Norton P, Zhuo D, Mason P, et al. Effectiveness of integrating delayed computed tomography angiography imaging for left atrial appendage thrombus exclusion into the care of patients undergoing ablation of atrial fibrillation. Heart Rhythm. 2016;13(1):12-9. https://doi.org/10.1016/j.hrthm.2015.09.002

10. Teunissen C, Habets J, Velthuis BK, Cramer MJ, Loh P. Double-contrast, single-phase computed tomography angiography for ruling out left atrial appendage thrombus prior to atrial fibrillation ablation. Int J Cardiovasc Imaging. 2017;33(1):121-8. https://doi. org/10.1007/s10554-016-0973-2

11. Dorenkamp M, Sohns C, Vollmann D, Lüthje L, Seegers J, Wachter R, et al. Detection of left atrial thrombus during routine diagnostic work-up prior to pulmonary vein isolation for atrial fibrillation: Role of transesophageal echocardiography and multidetector computed tomography. Int J Cardiol. 2013;163(1):26-33. https://doi.org/10.1016/j.jicard.2011.06.124

12. Tang RB, Dong JZ, Zhang ZQ, Li ZA, Liu XP, Kang JP, et al. Comparison of contrast enhanced 64-slice computed tomography and transesophageal echocardiography in detection of left atrial thrombus in patients with atrial fibrillation. J Interv Card Electrophysiol. 2008;22(3):199-203. https://doi.org/10.1007/s10840-008-9243-0

13. Hur J, Kim JY, Lee H-J, Nam JE, Ha J-W, Heo JH, et al. Dual-enhanced cardiac CT for detection of left atrial appendage thrombus in patients with stroke: a prospective comparison study with transesophageal echocardiography. Stroke. 2011;42(9):2471-7. https:// doi.org/10.1161/STROKEAHA.110.611293

14. Hur J, Kim YJ, Lee H-J, Nam JE, Hong YJ, Kim HY, et al. Cardioembolic stroke: dual-energy cardiac CT for differentiation of left atrial appendage thrombus and circulatory stasis. Radiology. 2012;263(3):688-95. https://doi.org/10.1148/radiol.12111691

15. Hur J, Kim YJ, Lee H-J, Ha J-W, Heo JH, Choi, E-Y, et al. Cardiac computed tomographic angiography for detection of cardiac sources of embolism in stroke patients. Stroke. 2009;40(6):2073-8. https://doi.org/10.1161/STROKEAHA.108.537928

16. Hur J, Kim YJ, Lee H-J, Ha J-W, Heo JH, Choi E-Y, et al. Left atrial appendage thrombi in stroke patients: detection with two-phase cardiac CT angiography versus transesophageal echocardiography. Radiology. 2009;251(3):683-90. https://doi.org/10.1148/ radiol.2513090794

17. Kim SC, Chun EJ, Choi SI, Lee S-J, Chang H-J, Han M-K, et al. Differentiation between spontaneous echocardiographic contrast and left atrial appendage thrombus in patients with suspected embolic stroke using two-phase multidetector computed tomography. Am J Cardiol. 2010;106(8):1174-81. https://doi.org/10.1016/j.amjcard.2010.06.033

18. Sawit ST, Garcia-Alvarez A, Suri B, Gaztanaga J, Fernandez-Friera L, Mirelis JG, et al. Usefulness of cardiac computed tomographic delayed contrast enhancement of the left atrial appendage before pulmonary vein ablation. Am J Cardiol. 2012;109(5):677-84. https://doi.org/10.1016/j.amjcard.2011.10.028

19. Hasegawa K, Miyazaki S, Ishida T, Tada H. Computed tomography in the prone position is a simple and useful technique to detect left atrial thrombi in persistent atrial fibrillation. J Cardiovasc Electrophysiol. 2018;29(4):632-3. https://doi.org/10.1111/jce.13411

20. Muramoto H, Higuchi K, Iwai S, et al. 1) Hiratsuka-Kyosai Hospital, Department of Cardiology, Kanagawa, Japan. 2) Hiratsuka-Kyosai Hospital, Department of Radiology. 3) Tokyo Medical and Dental University. Tokyo, Japan. HRS poster Session. May 2018.

21. Kantarci M, Ogul H, Sade R, Aksakal E, Colak A, Tanboga IH. Circulatory stasis or thrombus in left atrial appendage, an easy diagnostic solution. J Comput Assist Tomogr. 2019;43(3):406-9. https://doi.org/10.1097/RCT.0000000000000853

22. Kawaji T, Numamoto H, Yamagami S, Mabuchi R, Kitamura T, Enoki N, et al. Real-time surveillance of left atrial appendage thrombus during contrast computed tomography imaging for catheter ablation: THe Reliability of cOMputed tomography Beyond UltraSound in THROMBUS detection (THROMBUS) study. J Thromb Thrombolysis. 2019;47(1):42-50. https://doi.org/10.1007/s11239-018$1742-y$ 
23. Budoff MJ, Shittu A, Hacioglu Y, Gang E, Li D, Bhatia H, et al. Comparison of transesophageal echocardiography versus computed tomography for detection of left atrial appendage filling defect (Thrombus). Am J Cardiol. 2014;113(1):173-7. https://doi. org/10.1016/j.amjcard.2013.09.037

24. Singh N, Nallamothu N, Zuck V, Issa Z. Left atrial appendage filling defects on 64-slice multidetector computed tomography in patients undergoing pulmonary vein isolation: predictors and comparison to transesophageal echocardiography. J Comput Assist Tomogr. 2009;33(6):946-51. https://doi.org/10.1097/RCT.0b013e31819cabc3

25. Wu X, Wang C, Zhang C, Zhang Y, Ding F, Yan J. Systematic review/Meta-analysis: Computed tomography for detecting left atrial thrombus: a meta-analysis. Arch Med Sci. 2012;8(6):943-51._https://doi.org/10.5114/aoms.2012.32400

26. Kirchhof K, Welzel T, Mecke C, Zoubaa S, Sartor K. Differentiation of white, mixed, and red thrombi: value of CT in estimation of the prognosis of thrombolysis_phantom study. Radiology. 2003;228(1):126-30. https://doi.org/10.1148/radiol.2273020530

27. Bardo DME, Brown P. Cardiac multidetector computed tomography: basic physics of image acquisition and clinical applications. Curr Cardiol Rev. 2008;4(3):231-43. https://doi.org/10.2174/157340308785160615 